\title{
Kuntscher Nail: A Forgotten Entity Yet a Reliable Modality in Treatment of Winquist Type I and II Closed Femoral Shaft Fractures
}

Ajay Bharti ${ }^{1}$, Sanjay Kumar ${ }^{2}$, Sudhir Shyam Kushwaha ${ }^{1}$, Anil Kumar Gupta Sr. ${ }^{3,2}$, Nitish Kumar ${ }^{1}$, Atil K. $\mathrm{Lal}^{1}$

1. Department of Orthopedics, All India Institute of Medical Sciences, Gorakhpur, IND 2. Department of Orthopedics, Ganesh Shankar Vidyarthi Memorial Medical College, Kanpur, IND 3. Department of Orthopedics, Government Medical College Azamgarh, Azamgarh, IND

Corresponding author: Sanjay Kumar, drsk.gsvmortho@gmail.com

\section{Abstract \\ Background}

Interlocking intramedullary nail is used for almost all types of femoral shaft fractures worldwide because of its better mechanical stability. In countries like India with limited healthcare infrastructure, the use of Kuntscher nail (K-nail) in Winquist type I and type II isthmic fractures is still debated. Therefore, we conducted this study to compare the functional outcome, radiation exposure, and cost of the implant between closed reduction and internal fixation of Winquist type I and II fractures in the middle one-third shaft region by K-nail and intramedullary locked nails (IMILN), respectively.

\section{Methods}

This was a hospital-based non-blinded randomized trial which included 56 patients with closed Winquist type I and II femoral shaft fractures of the middle one-third femoral shaft (isthmic and paraisthmic zone). All the patients were either treated by K-nail or IMILN. The patients were followed up every three weeks for the initial six months and quarterly thereafter. Per operative duration of surgery and radiation exposure by Carm was documented and assuming scattered radiation $20 \%$, radiation exposure to the surgeon was calculated and patients were assessed clinically and radiologically for union.

\section{Results}

The patients were divided into two groups: group I (K-nail, $\mathrm{n}=25$ ) and group II (IMILN, $\mathrm{n}=31$ ). In groups I and II, the patients achieving radiological union were $88 \%(n=22 / 25)$ and $84 \%(n=27 / 31)$ at six months' duration, respectively. The mean duration for the radiological union was $3.65 \pm 0.55$ months in group I (Knail) and $3.76 \pm 0.59$ months in group II (IMILN), respectively. There was no statistically significant difference in the duration of the union $(\mathrm{p}=0.4963)$. The average number of $\mathrm{C}$ - arm exposures was $16.36 \pm$

Received 08/17/2020

Review began $08 / 29 / 2020$ Review ended 09/20/2020 Published 09/23/2020

\section{() Copyright 2020}

Bharti et al. This is an open access article distributed under the terms of the Creative Commons Attribution License CC-BY 4.0., which permits unrestricted use, distribution, and reproduction in any medium, provided the original author and source are credited.
3.18 in group I as compared to $27.29 \pm 4.01$ in group II, and the mean scattered radiation was $5.0 \pm 1.63$ in group I and $6.61 \pm 4.06$ in group II. This difference was statistically significant.

\section{Conclusion}

Kuntscher intramedullary nailing can provide comparable rates of union as is achieved with interlocking intramedullary nailing with an advantage of less radiation exposure and duration of surgery, provided the patient selection is proper (isthmic and paraisthmic zone).

Categories: Orthopedics, Trauma

Keywords: kuntscher nailing, interlocking nailing, winquist type i and ii, femoral shaft fractures

\section{Introduction}

Femoral shaft fractures are one of the commonest high energy fractures, and intramedullary nailing has now become the gold standard management of these fractures $[1,2]$. Intramedullary fixation is considered superior to plate fixation because of lower rates of infection, early weight-bearing, and lesser chances of non-union [3]. Gerhard Kuntscher in 1940 first introduced the cloverleaf nail for the intramedullary internal fixation of femoral shaft fractures [4]. Later, various studies [5,6] proved that unlocked Kuntscher nail (Knail) should only be used for isthmic femoral shaft fractures of Winquist type I and type II. The main drawback of K-nail for use in other types of femoral shaft fracture was lack of rotational stability. Nowadays, interlocking intramedullary nail is used for almost all types of femoral shaft fractures in developed countries because of better rotational stability.

However, in countries like India with limited healthcare infrastructure, the use of K-nail in Winquist type I 
and type II isthmic fractures is still debated. The reason for this includes lesser duration of the surgery, cost of the implant, lesser radiation exposure, or the procedure can be done without image intensifier also and has a comparable functional outcome with an interlocking nail (ILN) in these types of fractures.

We, therefore, conducted this study to compare the functional outcome, radiation exposure, and cost of the implant between closed reduction and internal fixation of Winquist [7] type I and II fractures in the middle one-third shaft region by K-nail and intramedullary locked nails (IMILN), respectively.

\section{Materials And Methods \\ Study design}

This is a hospital-based non-blinded randomized trial conducted in the Medical College of Northern India.

\section{Participants}

Patients between the age of 18 and 55 years with closed Winquist type I and II femoral shaft fractures of the middle one-third femoral shaft (isthmic and paraisthmic zone) reporting to the emergency department were included in the study. Patients with medical comorbidities, pathological fracture, fractures with vascular injury, open fractures, and those cases in which open reduction was needed were excluded from the study.

\section{Study duration and sample size}

Due to the variable number of patients reporting to the emergency with Winquist type I and II femoral shaft fractures of the middle one-third femoral shaft (isthmic and paraisthmic zone), no sample size was calculated. Instead, a study duration of three years was fixed, including 18 months of enrolment period (December 2013 and May 2015).

\section{Methodology}

All patients reporting to the emergency department were assessed for the nature and type of fracture according to a fixed protocol of the Institute. After diagnosis and written informed consent, abovementioned patients were included in the study. Those participants who agreed to participate in the study were randomly allocated to either one of the intervention groups stated above by concealed envelopes using the block randomization method.

\section{Intervention}

All the selected patients underwent closed reduction and internal fixation with intramedullary K-nailing (IMKN) group I and intramedullary interlocking nailing (IMILN) group II, as per the standard technique [7].

\section{Follow-up of patients}

Routinely all the patients were followed up every three weeks for an initial six months and quarterly thereafter. On each visit, patients were assessed clinically and radiologically for union and complications, if any. Partial weight-bearing was started in group I cases only after radiological evidence of bridging callus formation and full weight-bearing after fracture union. In group II, partial weight-bearing was started as soon as pain permitted followed by full weight-bearing after fracture union. The functional outcome was also assessed using the modified Harris hip score [8] and Knee society score [9].

\section{Outcome}

The mean duration of union (in months) was considered as the primary outcome. The functional outcome measured at nine months was considered as a secondary outcome.

\section{Study definitions}

The radiological union was defined as the presence of bridging callus across the fracture site in at least three cortices in both anteroposterior (AP) and lateral X-ray images [10].

The fracture was considered as healed or consolidated when there was complete radiological union and the patient was able to bear weight on his operated limb without pain at the fracture site [10].

The fractures were categorized in state of delayed union which took more than six months for union and those fractures in which there was an absence of callus at six months were in a state of non-union [6].

\section{Statistical analysis}

Data were entered in Microsoft excel and analysis was done using SPSS version 22 (Armonk, NY: IBM

Corp). T statistics for continuous data and chi-square test for categorical data were used, and p values of less 


\section{Cureus}

than 0.05 were considered statistically significant. Per protocol, analysis was performed. The functional outcome was also assessed using the modified Harris hip score and Knee society score according to the standard methods.

Approval was taken from the Institutional review board prior to the study (IEC/RC/Ortho/ 02/Thesis/Oct2013). Written informed consent was obtained from all the participants prior to the study. Confidentiality of the patient data was maintained throughout the study period.

\section{Results}

At the end of one and half year, 68 cases were enrolled in the study; out of which 9 cases were lost to followup and 3 cases required open reduction (group I); therefore, finally we had total 56 cases as per our inclusion criteria with an average follow-up of 18.08 months (range, 9-36 months).

Either of the two surgical procedures was performed on the patients as per simple random allocation. There were 25 patients in group I, who were managed by IMKN, and 31 patients in group II, who were managed by IMILN. The mean age of the patients was 34.28 years. There was no significant difference between both the groups in terms of sex and percentage of fracture type.

In groups I and II, the patients achieving radiological union were $88 \%(\mathrm{n}=22 / 25)$ and $84 \%(\mathrm{n}=27 / 31)$ at six months' duration, respectively. The mean duration for radiological union was $3.65 \pm 0.55$ months in group I (K-nail) and $3.76 \pm 0.59$ months in group II (IMILN), respectively (Table 1; Figures 1, 2).

\begin{tabular}{|c|c|c|c|}
\hline Procedure & No. of cases & Mean duration for union (months) & Mean duration for full weight-bearing (months) \\
\hline Group I( K-naling) & 25 & $3.65 \pm 0.55$ & $4.64 \pm 0.54$ \\
\hline Group II (interlocking nailing) & 31 & $3.76 \pm 0.59$ & $4.63 \pm 0.60$ \\
\hline Pvalue & & 0.478 & 0.949 \\
\hline
\end{tabular}

TABLE 1: Status of union and full weight-bearing in both groups of nailing

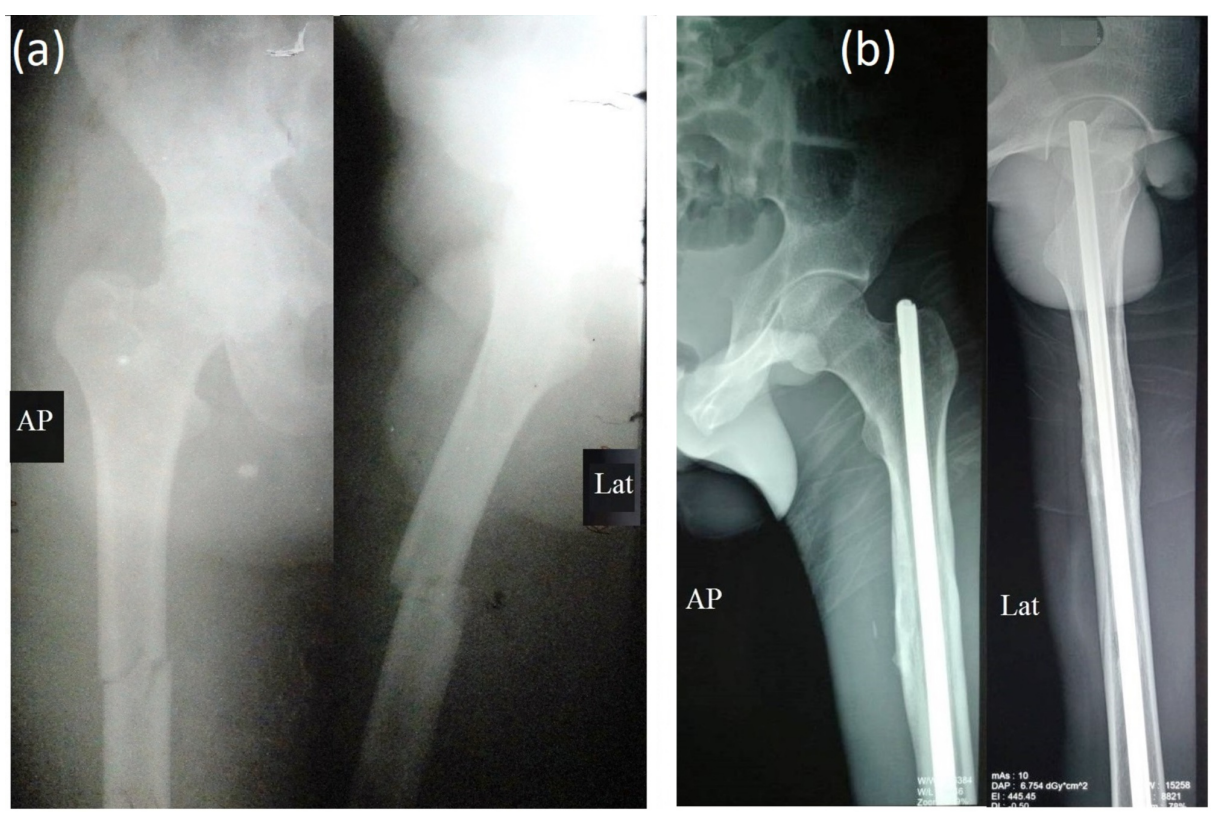

FIGURE 1: (a) Preop X-ray. (b) Postop X-ray at three years. Follow-up of a case managed with K-nailing (group I) 


\section{Cureus}
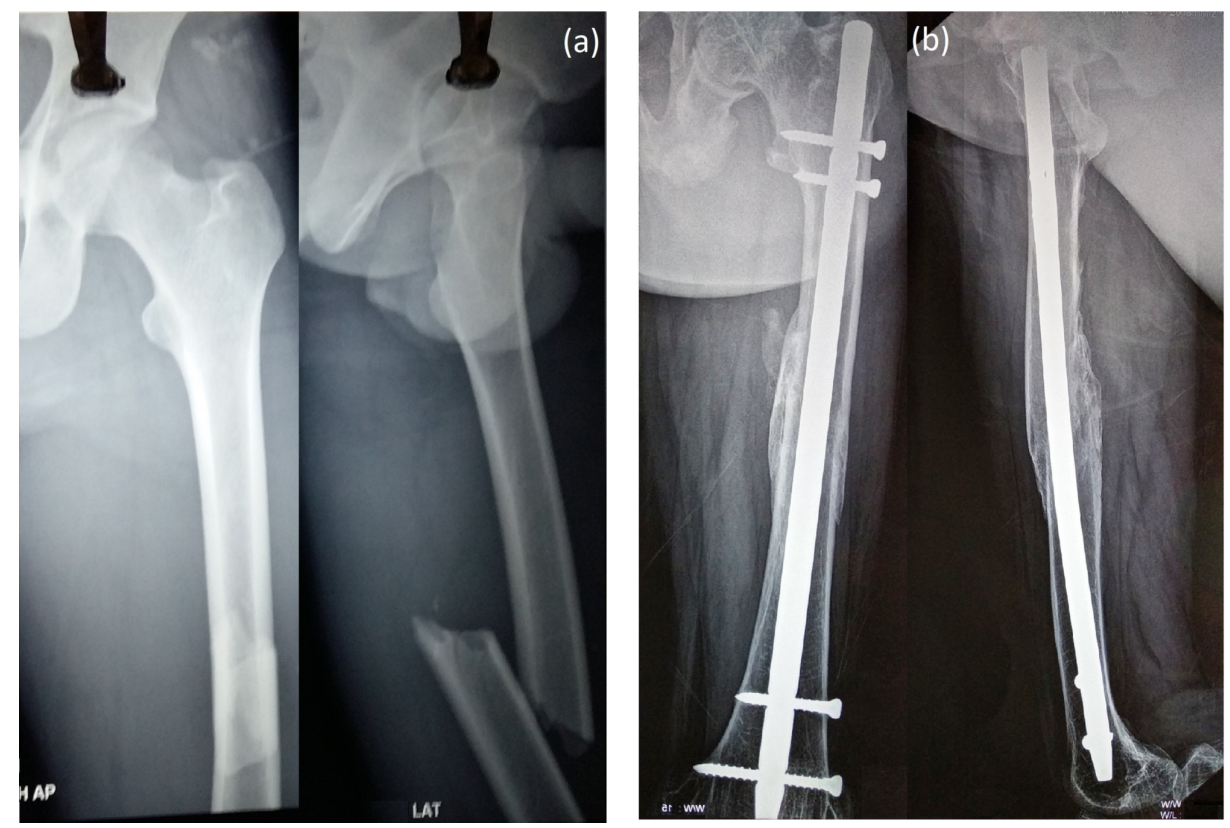

FIGURE 2: (a) Preop X-ray. (b) Postop X-ray at 22 months. Follow-up of a case managed with interlocking nailing (group II)

There was no statistically significant difference in the duration of union $(\mathrm{p}=0.4963)$. Functional outcome was assessed by the modified Harris hip score and Knee Society Score ( $p=0.8316$ and 0.525$)$ between the groups; both groups had more than $90 \%$ of its scores in the excellent to good range (Table 2).

\begin{tabular}{|c|c|c|}
\hline Modified Harris hip score & Group I( K-naliling) & Group II (interlocking naling) \\
\hline Excellent, $>90$ & 88\% (22) & 91\% (28) \\
\hline Good, 80-89 & $08 \%$ (2) & $03 \%$ (1) \\
\hline Satisfactory, 70-79 & $04 \%(1)$ & $06 \%$ (2) \\
\hline Poor, $<70$ & 00 & 00 \\
\hline \multicolumn{3}{|l|}{ Knee Society score } \\
\hline Excellent, 80-100 & $93 \%(23)$ & $87 \%(27)$ \\
\hline Good, $70-79$ & 00 & $03 \%$ (1) \\
\hline Fair, 60-69 & $08 \%$ (2) & $10 \%(3)$ \\
\hline Poor, $<60$ & 00 & 00 \\
\hline
\end{tabular}

TABLE 2: Functional outcome at latest follow-up with an average follow-up of 18.08 months (range 9-36 months) by the modified Harris hip score and Knee Society score

The mean duration of surgery was lower in group I ( $36.80 \pm 3.18)$ as compared to group II (50.32 \pm 7.12$)$, the average number of C-arm exposures was $16.36 \pm 3.18$ in group I as compared to $27.29 \pm 4.01$ in group II, and mean scattered radiation was $5.0 \pm 1.63$ in group I and $6.61 \pm 4.06$ in group II. All these differences were statistically significant (Table 3). 


\section{Cureus}

\begin{tabular}{|c|c|c|c|c|c|c|}
\hline $\begin{array}{l}\text { Intervention } \\
\text { groups }\end{array}$ & $\begin{array}{l}\text { No. of } \\
\text { cases }\end{array}$ & $\begin{array}{l}\text { Mean duration of } \\
\text { surgery in minutes }\end{array}$ & $\begin{array}{l}\text { Average number of } \mathrm{C} \text {-arm } \\
\text { radiation exposure }\end{array}$ & $\begin{array}{l}\text { Mean duration of C-arm radiation } \\
\text { exposure in seconds }\end{array}$ & $\begin{array}{l}\text { Mean C-arm radiation emitted in } \\
\text { millisievert (mSv) }\end{array}$ & $\begin{array}{l}\text { Mean C-arm scattered radiation exposure to } \\
\text { surgeon in millisievert (mSv) }\end{array}$ \\
\hline Group I (K-nailing) & 25 & $36.80 \pm 3.18$ & $16.36 \pm 3.18$ & $54 \pm 17.38$ & $25.18 \pm 8.12$ & $5.0 \pm 1.63$ \\
\hline $\begin{array}{l}\text { Group II } \\
\text { (interlocking } \\
\text { nailing) }\end{array}$ & 31 & $50.32 \pm 7.12$ & $27.29 \pm 4.01$ & $71.29 \pm 43.49$ & $33.25 \pm 20.30$ & $6.61 \pm 4.06$ \\
\hline $\begin{array}{l}\text { Statistical } \\
\text { significance }\end{array}$ & $\begin{array}{l}P \text { value } \\
<0.05\end{array}$ & Yes & Yes & Yes & Yes & Yes \\
\hline
\end{tabular}

TABLE 3: Per operative C-arm exposure and duration of surgery

Complications comprised of (Table 4, Figure 3) two cases of delayed union (4\%) with implant failure (bent nail) and one case of implant migration in group I and one case in this group developed superficial postoperative wound infection which resolved after a course of antibiotics with no further evidence of deeper infection or osteomyelitis and no further intervention was required.

\begin{tabular}{|c|c|c|}
\hline Complications & Group I (K-nailing) $(n=25)$ & Group II (interlocking nailing) $(n=31)$ \\
\hline Non-union & 00 & $6.4 \%$ (2) \\
\hline Delayed union & $08 \%$ (2) & $9.6 \%(3)$ \\
\hline Implant failure (Including implant migration) & $04 \%(1)$ & 00 \\
\hline Infection & 00 & 00 \\
\hline Pvalue & 0.192 & \\
\hline
\end{tabular}

TABLE 4: Postoperative complications associated with K-nailing and intramedullary interlock nailing
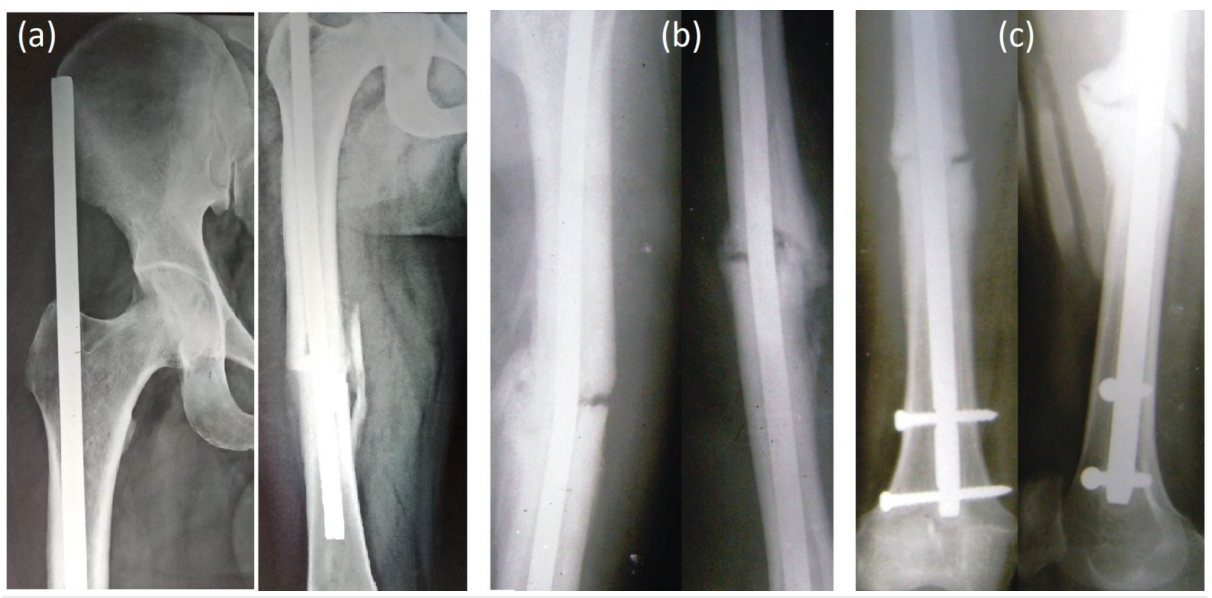

FIGURE 3: (a) Proximal migration of K-nail, (b) bent K-nail in group I. (c) Non-union in interlocking nailing in group II

All three complicated cases in group I were treated by exchange nailing with thicker nail, and eventually all of them proceeded to union. There were two cases of non-union and three cases of delayed union in group II. The patients with non-union in this group were successfully treated with exchange nailing with thicker nail with cancellous bone graft, while the three patients with delayed union were treated by mere dynamization, which eventually got united. There was no implant failure in group II patients. 


\section{Discussion}

Interlocking intramedullary nailing has now become the implant of choice for the treatment of almost all types of fractures of the femoral shaft. Most of the centers routinely use ILNs to fix all types of femoral shaft fractures, including fractures of isthmic and paraisthmic zone. The use of unlocked intramedullary nail (Knail) has virtually been abandoned.

Unlocked intramedullary nails were initially developed for fixation of transverse and short oblique fractures around the mid shaft region, i.e., the isthmic and paraisthmic areas of the shaft [11]. Nails with the diameter of the narrowest part of the medullary canal were usually used and they resisted rotational displacement by three-point fixation and friction. Interdigitation of the fracture ends provided further resistance against rotation. Later on, various studies proved the superiority of ILNs for shaft fractures except in the middle one-third shaft fractures. Therefore, K-nails were usually reserved for Winquist I and II femoral shaft fractures around the isthmus. The present study demonstrates that there is no significant difference in the outcomes between ILN and K-nail fixation of the patients when considering the union and the femoral alignment. The percentage of delayed union and non-union encountered in the group I and II was also not significant. Our major concern was the fact that group I patients had a higher rate of implant failure involving migration and bending of the nail. With our results, we concluded that the surgeons should probably reassess weight-bearing protocols when unlocked intramedullary nail fixation is employed and it seems that K-nail had an advantage over ILN when used for fracture around isthmus and paraisthmic area of diaphysis with the added advantage of less demanding surgery.

In our series, at sixth months we had union in $88 \%$ patients in group I and $84 \%$ patients in group II; patients in group I were walking without support by six months' duration (with an average of $4.64 \pm 0.54$ months), whereas unsupported average walking time in cases of group II was $4.63 \pm 0.60$ months $(p=0.946)$ (Table 1$)$.

We compared our results with studies of King et al. [12]. They reported 100\% union at six months in 112 cases treated by K-nail. The infection rate was $1.7 \%$. We had encountered two cases of non-union that were due to poor patient compliance. Similarly, Winquist et al. [13] reported union in $87 \%$ cases at three months amongst 520 cases and shortening of more than $2 \mathrm{~cm}$ in $20 \%$ cases managed by K-nail. Unsupported walking time was six weeks in 300 cases. Approximately $13 \%$ of cases landed in delayed union and non-union. Infection was reported in $9.6 \%$ of the cases. The results of their study are also comparable to the present series except for the infection which we did not encounter. The significant difference in unsupported walking time in our series was due to the reason that a large group of patients belonged to low socioeconomic status with less education. Therefore, it was judged that it is always better to wait for radiological fracture union before protected weight-bearing was allowed. Shortening was not a frequent complication encountered except in few cases $(1-2.5 \mathrm{~cm})$ of Winquist type II fractures: group I, three cases and group II, one case. We did not encounter any impacted nail, transient common peroneal nerve palsy, fat embolism, or any pulmonary complications in our cases.

Yu et al. [6] in their study of 93 cases of intramedullary nail fixation (43 cases using open K-nail and 50 with ILN) stated that there was no difference in the union rate between both groups $(p=0.3282)$. There were two cases of non-union and one case of delayed union in the K-nail group. There was one case of non-union and two cases of delayed union in the ILN group, which is comparable with the present study.

As a healthcare provider, our major concern is of exposure to ionizing radiation during surgery which is encountered as direct and scattered radiation from Image intensifier. Hak in his study stated that scattered radiation is the main source of radiation exposure for surgeons as well as for the patient. Out of around 1,000 photons emitted by intensifier, only 20 photons reach the detector, $100-200$ photons, i.e. around $20 \%$ radiation, are scattered, and remaining photons are absorbed by the patient. Adverse effects of radiation on the body can be due to either somatic effects or stochastic effects. Somatic effects are directly related to the radiation dose. The dose of radiation required to produce radiation sickness is between 500 and 1,000 mSv. Early somatic effects include radiation sickness, whereas late somatic effects include leukemia, thyroid cancer, and radiation-induced cataracts so radiation exposure should be kept as low as reasonably achievable during nailing [14]. Fuchs et al. in their study found that during eight cases of closed ILN the average radiation dose received by the eye was $0.019 \mathrm{mSv}$, by the thyroid gland was $0.0354 \mathrm{mSv}$, and by the hands was $0.0417 \mathrm{mSv}$ [15]. Our study clearly states that in comparison to K-nail, IMILN is associated with more radiation (Table 3) and maximum exposure to radiation occurs during distal locking [16]. With the advent of electromagnetic-assisted technique, radiation during distal locking has been effectively reduced in comparison to freehand fluoroscopy-assisted technique; moreover, electromagnetic-assisted technique is costly and also requires proper training in electromagnetic-assisted surgical techniques [17].

Thus, the complexity of implant and technique of IMILN increases the duration of surgery and radiation exposure. Therefore, they should also be given due importance and K-nail should be preferred over IMILN. As per the Indian scenario, we also found that K-nailing procedure is very cost-effective as we calculated the difference of financial burden on patients. The mean overall expenditure in K-nailing came out to be $1679 \pm$ 0.80 Indian rupee, while it was $5032 \pm 0.69$ Indian rupee in ILN that was also statistically significant $(\mathrm{p}<$ $0.05)$. 


\section{Limitation of the study}

The limitations in our study were small sample size and non-availability of radiation dosimeter to record scattered radiation from image intensifier.

\section{Conclusions}

Kuntscher intramedullary nailing can provide comparable rates of union as is achieved with interlocking intramedullary nailing with an advantage of less radiation exposure and duration of surgery, provided the patient selection is proper (isthmic and paraisthmic zone). There is however a higher incidence of implant migration and bending and hypertrophic non-union associated with the use of K-nailing which occurred due to improper implant size selection. Considering the cost and surgical aspects and functional outcome, K-nail is still a viable option for a majority number of cases in many Indian hospitals, especially those with limited financial resources or less technical expertise.

\section{Additional Information \\ Disclosures}

Human subjects: Consent was obtained by all participants in this study. Institutional Review Board GSVM Medical College, Kanpur issued approval IEC/RC/Ortho/ 02/Thesis/Oct2013. This study to compare the functional outcome, radiation exposure and cost of the implant between closed reduction and internal fixation of Winquist type I and II fractures of in middle one-third shaft region by K-nail and intramedullary locked nails has been approved by IRB of GSVM Medical College, Kanpur, UP, India. Animal subjects: All authors have confirmed that this study did not involve animal subjects or tissue. Conflicts of interest: In compliance with the ICMJE uniform disclosure form, all authors declare the following: Payment/services info: All authors have declared that no financial support was received from any organization for the submitted work. Financial relationships: All authors have declared that they have no financial relationships at present or within the previous three years with any organizations that might have an interest in the submitted work. Other relationships: All authors have declared that there are no other relationships or activities that could appear to have influenced the submitted work.

\section{Acknowledgements}

We thank Dr. Tanu Midha, Associate Professor, Department of Community Medicine, GSVM Medical College Kanpur for her sincere efforts related to statistical analysis of this article.

\section{References}

1. Denisiuk M, Afsari A: Femoral Shaft Fractures. StatPearls Publishing, Treasure Island, FL; 2020.

2. Testa G, Vescio A, Aloj DC, et al.: Definitive treatment of femoral shaft fractures: comparison between anterograde intramedullary nailing and monoaxial external fixation. J Clin Med. 2019, 8:1119. $10.3390 /$ jcm8081119

3. Babalola OM, Ibraheem GH, Ahmed BA, Olawepo A, Agaja SB, Adeniyi A: Open intramedullary nailing for segmental long bone fractures: an effective alternative in a resource-restricted environment. Niger J Surg. 2016, $22: 90-95.10 .4103 / 1117-6806.188983$

4. Kuntscher G: Die marknagelung von knochenbruchen. Arch Klin Chir. 1940, 200:443-455.

5. Ling HT, Ng WM, Kwan MK, Aizuddeen LKF, Tay PCM: Use of unlocked intramedullary nailing in winquist type I and II femoral isthmus fracture. Malays Orthop J. 2008, 2:17-22.

6. Yu CK, Wong HY, Vivek AS, Se To BC: Unlocked nailing vs. interlocking nailing for winquist type I and II femoral isthmus fractures. Is there a difference?. Malays Orthop J. 2008, 2:23-27.

7. Canale ST, Beaty J: Campbell's Operative Orthopaedics, 12th Edition. Elsevier/Mosby, St. Louis, MO; 2012.

8. Vishwanathan K, Akbari K, Patel AJ: Is the modified Harris hip score valid and responsive instrument for outcome assessment in the Indian population with per trochanteric fractures?. J Orthop. 2018, 15:40-46. 10.1016/j.jor.2017.12.001

9. Knee society score. (2019). Accessed: December 12, 2019: https://orthopaedicscore.com/scorepages/knee_society_score.html.

10. Ricci WM, Bellabarba C, Evanoff B, Herscovici D, Di Pasquale T, Sanders R: Retrograde versus antegrade nailing of femoral shaft fractures. J Orthop Trauma. 2001, 15:161-169. 10.1097/00005131-200103000-00003

11. Schatzker J, Tile M: Fractures of the femur. The Rationale of Operative Fracture Care. Springer, 1996. 367385.

12. King KF, Rush J: Closed intramedullary nailing of femoral shaft fractures. A review of one hundred and twelve cases treated by the Kuntscher technique. J Bone Joint Surg Am. 1981, 63:1319-1323.

13. Winquist RA, Hansen ST Jr, Clawson DK: Closed intramedullary nailing of femoral fractures. A report of five hundred and twenty cases. J Bone Joint Surg Am. 1984, 66:529-539.

14. Hak DJ: Radiation exposure during intramedullary nailing. Injury. 2017, 48:S26-S29. 10.1016/j.injury.2017.04.023

15. Fuchs M, Schmid A, Eiteljorge T, Modler M, Stürmer KM: Exposure of the surgeon to radiation during surgery. Int Orthop. 1988, 22:153-156. 10.1007/s002640050230

16. Grimwood D, Harvey-Lloyd J: Reducing intraoperative duration and ionising radiation exposure during the insertion of distal locking screws of intramedullary nails: a small-scale study comparing the current fluoroscopic method against radiation-free, electromagnetic navigation. Eur J Orthop Surg Traumatol. 2016, 26:867-876. 10.1007/s00590-016-1835-2 


\section{Cureus}

17. Kobayashi H, Tanikawa H, Ogawa R, Okuma K, Kobayashi S, Harato K, Niki Y: Site-specific radiation exposure during intramedullary nails: a comparison between the free-hand technique and the electromagnetic assisted technique. Biomed J Sci Tech Res. 2018, 3:3233-3237. 